\title{
Special issue editorial: Sequences and their applications
}

\author{
Tor Helleseth • Jonathan Jedwab
}

Received: 4 January 2014 Published online: 16 January 2014

(C) Springer Science+Business Media New York 2014

We are very pleased to introduce this special issue on Sequences and their Applications. Sequences (and arrays, their higher-dimensional relatives) exhibit a strong interplay between theory and application. In one direction, the study of sequences has benefitted greatly from motivating applications in communications, cryptography, coding, and combinatorics. In the other direction, the development of the theory of sequences using tools from finite fields, finite geometry, analysis and number theory has enabled the solution of practical design problems in emerging applications. The purpose of this special issue is to highlight advances in both the theory and application of sequences.

The invited paper "A complementary construction using mutually unbiased bases" by $\mathrm{G}$. Wu and $\mathrm{M}$. Parker introduces a previously unrecognized connection between the construction of pairs of (Golay) complementary sequences and arrays, that are particularly valuable in wireless communications, and the mutually unbiased bases of quantum information theory. This novel point of view opens up new directions for research into complementary sequences.

The paper "A simple suboptimal construction of cross-bifix-free codes" by D. Bajic and T. Loncar-Turukalo concerns the construction of sets of codewords (sequences) that are suitable for communications synchronization, in the sense that no codeword ends the way another one starts. The paper has both expository and historical value: an earlier version influenced the development of international mobile communications standards as a research report in 2007 , but until now its contents have not been made publically available.

\footnotetext{
T. Helleseth $(\bowtie)$

Department of Informatics, University of Bergen, PB 7803, 5020 Bergen, Norway e-mail: Tor.Helleseth@ii.uib.no

J. Jedwab

Department of Mathematics, Simon Fraser University, 8888 University Drive, Burnaby, BC V5A 1S6, Canada

e-mail: jed@sfu.ca

T. Helleseth

Department of Informatics, Høgteknologisenteret, Thormøhlensgate 55, 5008 Bergen, Norway
} 
The paper "New near perfect sequences of even lengths" by R. Hariharan and T.E. Hall uses a finite field to construct near perfect sequences having applications in channel estimation and radar. The construction results in infinite families of near perfect sequences of new even length, subject to the correctness of a conjecture presented in the paper.

The paper "Perfect arrays of unbounded sizes over the basic quaternions" by S. Barrera Acevedo and N. Jolly shows the existence of perfect arrays of unbounded sizes over the alphabet $\{1,-1, i,-i, j,-j, k,-k\}$. They show the existence of perfect arrays for all sizes $2 p \times n p$, where $2 n=p+1 \equiv 2(\bmod 4), p$ is a prime number and $n$ is odd.

The paper "Paraunitary generation/correlation of QAM complementary sequence pairs" by S.Z. Budišin and P. Spasojević presents a decomposition of complementary sequence pairs with arbitrary alphabet and length based on paraunitary matrices. A length $N$ complementary pair can be recursively constructed using such matrices. The constructions of rectangular and hexagonal QAM complementary sequences are discussed for various constellations. The new methods that are described to construct complementary pairs with any length $N$ can be useful for practical applications. 